\title{
Low Temperature Thermal Expansion of Cubic Fullerene $\mathrm{C}_{60}$
}

\author{
Vinu T. $\mathbf{P}^{1}$, Sreelatha $\mathbf{K}^{2}$ \\ NSS Hindu College, Changanasserry, Kottayam, Kerala, India-686 102
}

\begin{abstract}
The anisotropy in the low temperature thermal expansion of $C_{60}$ is analyzed theoretically. The generalized Grïneisen parameters (Gps) of the elastic waves propagating in different directions in $C_{60}$ are calculated using the second-order elastic constants and the first-order pressure derivatives of the second-order elastic constants. The expressions of effective second-order elastic constants are used to evaluate the six third-order elastic constants of the $C_{60}$ system. The third-order elastic constants of $C_{60}$ thus obtained are negative. The values of generalized Grüneisen parameters are positive. The Brugger gamma is calculated and the low-temperature limit of Grüneisen gamma is determined using the procedure of Menon and Ramji Rao. The value of low-temperature limit of Grüneisen gamma is positive. Therefore we expect volume expansion to be positive to absolute zero for $C_{60}$ system. The anisotropy of thermal expansion along the three axis of crystal is less.
\end{abstract}

PACS number(s): $62.20 \mathrm{D}, 74.25 \mathrm{~L}, 74.62$

Keywords: Thermal Expansion, third-order elastic constants, Grüneisen parameters

\section{Introduction}

$\mathrm{C}_{60}$ molecule, which has icosaheadral symmetry[1], has a structure of soccer ball with the $\mathrm{C}$ atoms situated at each vertex, forms a close-packed molecular solid [2]. At room temperature $\mathrm{C}_{60}$ crystallizes in FCC structure with space group $\mathrm{Fm} \overline{3} \mathrm{~m}$ [3]. The unit cell of $\mathrm{C}_{60}$ contains four molecules. Fullerenes are stable, cage like molecules that constitute the third form of carbon; the other two forms are diamond and graphite. This is a solid characterized by equal angles and all faces in the regular polygon. Since the discovery of the method for producing bulk quantities of $\mathrm{C}_{60}$, fullerene science has spread out rapidly in to solid-state physics, molecular physics, chemical sciences and material sciences [4].

The elastic moduli, thermodynamic potential, specific heat and the Grüneisen parameters are some of the fundamental characteristics of a solid. The study of these properties is essential to the understanding of $\mathrm{C}_{60}$ [5-12]. The discovery of carbon nanotubes [13] and the new method of producing them stimulates great interest in these materials [14]. The Grüneisen parameter [Gp], is one of the several methods of determining the anharmonicity in a solid [15].

The present objective is to study the thermal expansion and the Grüneisen parameters of $\mathrm{C}_{60}$. We have calculated the generalized isothermal Grüneisen parameters $\gamma_{j}$ for acoustic modes. This is achieved by means of the third-order elastic constants of $\mathrm{C}_{60}$ obtained from second-order elastic constants and first-order pressure derivatives of secondorder elastic constants. The mode Grüneisen gammas for $\mathrm{C}_{60}$ can be derived from the effective second-order elastic constant expressions.

\section{Theory}

\subsection{Third-order Elastic constants of $C_{60}$}

Third-order elastic constants quantify the lowest order anharmonic properties of solids such as thermal expansion, temperature and pressure dependence of elastic constants and interactions of acoustic and thermal phonons. The thirdorder elastic constants can be determined using the finite strain elasticity theory of Murnaghan [16], where the elastic stress is nonlinear with elastic strain. From the higher order elastic constants, on the basis of continuum approximation, it is possible to calculate the scattering of phonons with the aid of nonlinear elasticity theory. The expression for the effective second-order elastic constants and its pressure derivatives for the strained crystal in cubic system are given by Ramji Rao and Rajput [17]. The pressure derivatives of the second-order elastic constants are in terms of higher order elastic constants up to third-order. As this $\mathrm{C}_{60}$ has 3 independent second-order elastic constant, we have obtained 3 equation of effective second-order elastic constant in terms of strain component. We have calculated the complete set of 6 independent third-order elastic constants and they are presented in Table 1. All the third-order values obtained are negative.

\subsection{Low-temperature thermal expansion of $\mathrm{C}_{60}$}

The linear thermal expansion coefficients denoted by $\alpha$ can be expressed as

$$
\begin{aligned}
& \mathrm{V} \alpha=\left[\left(\mathrm{S}_{11}+2 \mathrm{~S}_{12}\right) \bar{\gamma}_{\mathrm{L}}(\mathrm{T})\right] \mathrm{C}_{\mathrm{v}} \\
& =\gamma^{\mathrm{Br}} \mathrm{C}_{\mathrm{v}} \chi_{\text {iso }}(1)
\end{aligned}
$$

Here $\mathrm{S}_{\mathrm{ij}}$ are the elastic compliance coefficients, $\mathrm{V}$ is the molar volume, $\mathrm{C}_{\mathrm{v}}$ is the molar specific heat in the $\mathrm{T}^{3}$ region, and $\chi_{\text {iso }}$ is the isothermal compressibility. $\bar{\gamma}(\mathrm{T})$ are the low- 


\section{International Journal of Science and Research (IJSR) \\ ISSN (Online): 2319-7064}

Index Copernicus Value (2013): 6.14 | Impact Factor (2014): 5.611

temperature limits of the effective Grüneisen functions. $\gamma^{\mathrm{Br}}$ is the average or effective Grüneisen functions in the notations of Brugger and Fritz [18]. The effective Grüneisen function can be defined as

$$
\bar{\gamma}(\mathrm{T})=\left[\sum_{\mathrm{qj}} \gamma(\mathrm{q}, \mathrm{j}) \mathrm{C}_{\mathrm{v}}(\mathrm{q}, \mathrm{j})\right]\left[\sum_{\mathrm{qj}} \mathrm{C}_{\mathrm{v}}(\mathrm{q}, \mathrm{j})\right]^{-1}
$$

These effective Grüneisen function is weighted averages of the generalized Grüneisen parameters $\gamma(q, j)$. q is the wave vector and $\mathrm{j}$ is the polarization index. $\mathrm{C}_{\mathrm{v}}(\mathrm{q}, \mathrm{j})$ is the contribution of a single normal mode of frequency $\omega$, wave vector $q$ and polarization index $\mathrm{j}$ to the specific heat of the lattice $\gamma(\mathrm{q}, \mathrm{j})$ is given by

$$
\bar{\gamma}(\mathrm{q}, \mathrm{j})=-\frac{\partial \log \omega(\mathrm{q}, \mathrm{j})}{\partial \log \varepsilon}
$$

Where $\varepsilon$ is a uniform and strain in the Bessel plane. Here, instead of choosing arbitrary strain components in defining Gps, we choose such strain that leave the symmetry of crystal unchanged. Here we use the Langragian strain parameter $\mathrm{n}_{\mathrm{ij}}$, which are in place of volume change, are selected such that $\mathrm{i}=\mathrm{j}$ (where $\mathrm{I}=\mathrm{j}=1,2,3$ ), in the quasiharmonic approximation.

At low temperature, the Gps of the elastic wave frequencies determine the anisotropy thermal expansion of a uniaxial

$$
\rho_{0} \omega^{2} u_{i}=4 \pi^{2} \sum_{k j l} u_{j} Y_{l} Y_{k}\left\lfloor C_{i k, j l}+\sum_{m n} \varepsilon_{m n}\left(C_{i k, j l, m n}+C_{i k, n l} \delta_{m j}+C_{n k, j l} \delta_{m i}+C_{k l, m n} \delta i j\right)\right\rfloor
$$

Here $\mathrm{Y}_{\mathrm{i}}$ 's are the component of the arbitrary direction in which the wave is propagating, $u_{i}$ 's the components of displacement. $\omega$ is the angular frequency and $\rho_{0}$ is the density of the crystal in the unstrained state. The $\mathrm{C}_{\mathrm{ij}, \mathrm{kl}}$ and $\mathrm{C}_{\mathrm{kl}, \mathrm{mn}}$ values are the second-order and third-order elastic constants respectively. From the elements of the secular determinant formed by the coefficients of $u_{i}$ 's, the expression for the effective second-order elastic constant in terms of the strain component $\varepsilon$ are obtained as

$$
\begin{aligned}
& \mathrm{C}_{11}^{\prime}=\mathrm{C}_{11}+\left[\left(\mathrm{C}_{111}+2 \mathrm{C}_{112}+3 \mathrm{C}_{11}+2 \mathrm{C}_{12}\right) \frac{\varepsilon^{\prime}}{3}\right] \\
& \mathrm{C}_{12}^{\prime}=\mathrm{C}_{12}+\left[\left(2 \mathrm{C}_{112}+\mathrm{C}_{123}-\mathrm{C}_{11}\right) \frac{\varepsilon^{\prime}}{3}\right] \\
& \mathrm{C}_{44}^{\prime}=\mathrm{C}_{44}+\left[\left(2 \mathrm{C}_{155}+\mathrm{C}_{144}+2 \mathrm{C}_{12}+\mathrm{C}_{11}+2 \mathrm{C}_{44}\right) \frac{\varepsilon^{\prime}}{3}\right]
\end{aligned}
$$

Here the $\mathrm{C}_{\mathrm{ij}}$ and $\mathrm{C}_{\mathrm{ijk}}$ are, respectively, the second-order and third-order elastic stiffness constants in Voigt notations.

The general expressions for $\mathrm{Gp}$ from the determinantal Eq. (4) are

$$
\gamma_{i}=\frac{-1}{2 \bar{X}_{j}}\left\{\frac{\left\lfloor\bar{X}_{j} \frac{\partial}{\partial \varepsilon^{\prime}}(\mathrm{A}+\mathrm{B})\right\rfloor-\left\lfloor\frac{\partial}{\partial \varepsilon^{\prime}}\left(\mathrm{AB}-\mathrm{C}^{2}\right)\right\rfloor}{\left[2 \overline{\mathrm{X}}_{\mathrm{j}}-\left(\overline{\mathrm{A}}_{0}+\overline{\mathrm{B}}_{0}\right)\right]}\right\}
$$

crystal and the effective Grüneisen functions approach the limits

$$
\bar{\gamma}(-3)=\left[\int \sum_{\mathrm{j}=1}^{3} \gamma_{\mathrm{i}}(\theta, \varphi) \mathrm{V}_{\mathrm{i}}^{-3}(\theta, \varphi) \mathrm{d} \Omega\right]^{-1}
$$

Here $\mathrm{V}_{\mathrm{j}}(\theta, \varphi)$ is the wave velocity of elastic wave of polarization index $\mathrm{j}$, propagating in the direction $(\theta, \varphi)$. $\gamma_{\mathrm{j}}$ is the Gps of this acoustic mode. The Grüneisen parameter for the acoustic modes can be calculated using the second-order elastic constants and third-order elastic constants and hence the low temperature limits of Gps can be obtained from the procedure of Menon and Ramji Rao [19]. In cubic crystal, it is assumed that Gps and acoustic wave velocities depend only on $\theta$ and are independent of the azimuth $\varphi$.

The average effective Grüneisen function in the notations of Brugger and Fritz in equation (1) are expressed as

$\gamma^{\mathrm{Br}}=\left[\left(\mathrm{S}_{11}+2 \mathrm{~S}_{12}\right) \gamma\right] \chi_{\text {iso }}^{-1}$

\subsection{The mode gruneisen gammas of $\mathbf{C}_{60}$ system}

The general Thurston and Brugger equation for wave propagation is [20]

where

$\mathrm{A}=\mathrm{C}_{11}^{\prime} \sin ^{2} \theta+\mathrm{C}_{44}^{\prime} \cos ^{2} \theta$,

$\mathrm{B}=\mathrm{C}_{44}^{\prime} \sin ^{2} \theta+\mathrm{C}_{11}^{\prime} \cos ^{2} \theta$,

$\mathrm{C}=\sin \theta \cos \theta\left(\mathrm{C}_{12}^{\prime}+\mathrm{C}_{44}^{\prime}\right)$

and

$\mathrm{X}_{\mathrm{j}}=\rho_{0} \mathrm{~V}_{\mathrm{j}}^{2}(\theta, \varphi)$

Here we made use of the calculated values of $\mathrm{C}_{\mathrm{ij}}$ and $\mathrm{C}_{\mathrm{ijk}}$ (given in Table 1) for the calculation of $\gamma_{j}$. The wave velocities and the Gps for the elastic wave propagating at different angles $\theta$ to the axis of $\mathrm{C}_{60}$ are given in Table 2 . Fig. 1 gives the plot in polar coordinates of the generalized Grüneisen parameters $\gamma_{j}$ for acoustic mode as a function of angle $\theta$, which the direction of propagation makes with the crystal axis.

\section{Results and Discussions}

The low temperature limit of the Grüneisen parameters obtained in the present work for the cubic compound $\mathrm{C}_{60}$ have been compared with available results. An important feature of the Grüneisen parameter in $\mathrm{C}_{60}$ is that all the mode 


\section{International Journal of Science and Research (IJSR) \\ ISSN (Online): 2319-7064}

Index Copernicus Value (2013): 6.14 | Impact Factor (2014): 5.611

gammas are positive. Fig. 1 shows the variation of generalized Grüneisen parameters $\gamma_{\mathrm{j}}(\theta)$ as a function of angle $\theta$ for $\mathrm{C}_{60}$. The generalized Grüneisen parameter for elastic waves propagating at different angles $\theta$ for the crystal axis in the $\mathrm{C}_{60}$ system is summarized in Table 2. The acoustic mode of Grüneisen parameter $\gamma_{1}$ assumes a minimum value of 3.32 at the both angles $\theta=0$ and $90^{\circ}$. It has a maximum value of 3.43 at $\boldsymbol{\theta}=45^{\circ}$. While the acoustic mode $\gamma_{2}$ assumes a minimum value of 0.54 , at $\theta=$ $45^{\circ}$ and a maximum value of 3.35 at both the angles $\theta=0$ and $90^{\circ} .3 .35$ is the maximum value of $\gamma_{3}$ obtained both at $\theta=0$ and $90^{\circ}$ and has a minimum of 2.95 obtained at $\boldsymbol{\theta}=$ $45^{\circ}$. It is seen that the transverse acoustic modes and longitudinal acoustic modes are equal in the case of this cubic crystal $\mathrm{C}_{60}$.

Rao and Venkatesh [3] have estimated the Grüneisen parameter using the elastic constants of $\mathrm{C}_{60}$. Rao and Venkatesh [3] also calculated the second Grüneisen parameters $\gamma_{\mathrm{L}}$ as 3.3, which are given in Table 3. White et al.[15] calculated the over all Grüneisen parameter as 3, using the relationship between the frequency of the mode $\omega_{\mathrm{i}}$ and the volume $\mathrm{V}$, using the relation $\gamma_{\mathrm{i}}=\frac{-\delta \log \omega_{\mathrm{i}}}{\delta \log \mathrm{V}}$

The result obtained in the present work for the low temperature limit of lattice thermal expansion for cubic $\mathrm{C}_{60}$ is $\gamma_{\mathrm{L}}=2.8$, which is in good agreement with the results obtained by Rao and Venkatesh [21] and White et al [15]. For $\mathrm{C}_{60}$ we observe that at low temperatures the $\mathrm{C}_{60}$ molecules show a high degree of anharmonicity. This causes the deviation of the anharmonicity. The high degree of anharmonicity in $\mathrm{C}_{60}$ shows that $\mathrm{C}_{60}$ molecules have an incompressible hard core.

\section{Conclusion}

The mode Grüneisen parameters of $\mathrm{C}_{60}$ for different acoustic wave propagation direction have been calculated. The results show that the thermal expansion anisotropy of the material is less for various acoustic modes. The average Grüneisen function $\gamma^{\mathrm{Br}}$ is 0.94 . The Grüneisen parameter studies provide insight into the more or less isotropic nature in the thermal of $\mathrm{C}_{60}$. The low-temperature Grüneisen gamma is positive, so we expect volume expansion to be positive down to $0 \mathrm{~K}$ for $\mathrm{C}_{60}$.

Table 1: Second-order and third-order elastic constants of $\mathrm{C}_{60}$ in units of $\mathrm{GPa}$

\begin{tabular}{|lccccccccc|}
\hline Reference & $\mathrm{C}_{11}$ & $\mathrm{C}_{12}$ & $\mathrm{C}_{44}$ & $\mathrm{C}_{111}$ & $\mathrm{C}_{112}$ & $\mathrm{C}_{155}$ & $\mathrm{C}_{123}$ & $\mathrm{C}_{144}$ & $\mathrm{C}_{456}$ \\
\hline Present Work & 14.9 & 6.2 & 6.2 & -624 & -162 & -162 & -90 & -90 & -90 \\
\hline
\end{tabular}

Table 2: Generalized Grüneisen functions for elastic waves propagating at different angles $\theta$ for the crystal axis in the $C_{60}$ system

\begin{tabular}{|c|c|c|c|c|c|c|}
\hline $\boldsymbol{\theta}$ & $\boldsymbol{\gamma}_{\mathbf{1}}$ & $\boldsymbol{\rho}_{\mathbf{0}} \mathbf{v}_{\mathbf{1}}{ }^{\mathbf{1}}$ & $\boldsymbol{\gamma}_{\mathbf{2}}$ & $\boldsymbol{\rho}_{\mathbf{0}} \mathbf{v}_{\mathbf{2}}{ }^{\mathbf{2}}$ & $\boldsymbol{\gamma}_{\mathbf{3}}$ & $\boldsymbol{\rho}_{\mathbf{0}} \mathbf{v}_{\mathbf{3}}{ }^{\mathbf{2}}$ \\
\hline 0 & 3.32 & 14.9 & 3.35 & 6.2 & 3.35 & 6.2 \\
\hline 5 & 3.33 & 15.0 & 3.27 & 6.2 & 3.34 & 6.1 \\
\hline 15 & 3.36 & 15.4 & 2.65 & 6.2 & 3.25 & 5.6 \\
\hline 25 & 3.40 & 16.0 & 1.70 & 6.2 & 3.12 & 5.0 \\
\hline 35 & 3.42 & 16.5 & 0.87 & 6.2 & 3.00 & 4.5 \\
\hline 45 & 3.43 & 16.7 & 0.54 & 6.2 & 2.95 & 4.3 \\
\hline 55 & 3.42 & 16.5 & 0.87 & 6.2 & 3.00 & 4.5 \\
\hline 65 & 3.40 & 16.0 & 1.70 & 6.2 & 3.12 & 5.0 \\
\hline 75 & 3.36 & 15.4 & 2.65 & 6.2 & 3.25 & 5.6 \\
\hline 85 & 3.33 & 15.0 & 3.27 & 6.2 & 3.34 & 6.1 \\
\hline 90 & 3.32 & 14.9 & 3.35 & 6.2 & 3.35 & 6.2 \\
\hline
\end{tabular}

Table 3: The values of the Grüneisen parameters $\gamma^{\mathrm{Br}}$ and $\gamma_{L}$ for $\mathrm{C}_{60}$

\begin{tabular}{|c|c|c|c|}
\hline$\gamma^{\mathrm{Br}}$ & $\gamma_{L}$ & $\begin{array}{c}\text { Rao and } \\
\text { Venkatesh }^{3}\end{array}$ & \multirow{2}{*}{ White $^{15}$} \\
\cline { 1 - 2 } Present work & Present work & $\mathbf{3 . 3}$ & $\mathbf{3}$ \\
\hline $\mathbf{0 . 9 4}$ & $\mathbf{2 . 8}$ & $\mathbf{3 . 3}$ & \\
\hline
\end{tabular}




\section{International Journal of Science and Research (IJSR) \\ ISSN (Online): 2319-7064}

Index Copernicus Value (2013): 6.14 | Impact Factor (2014): 5.611

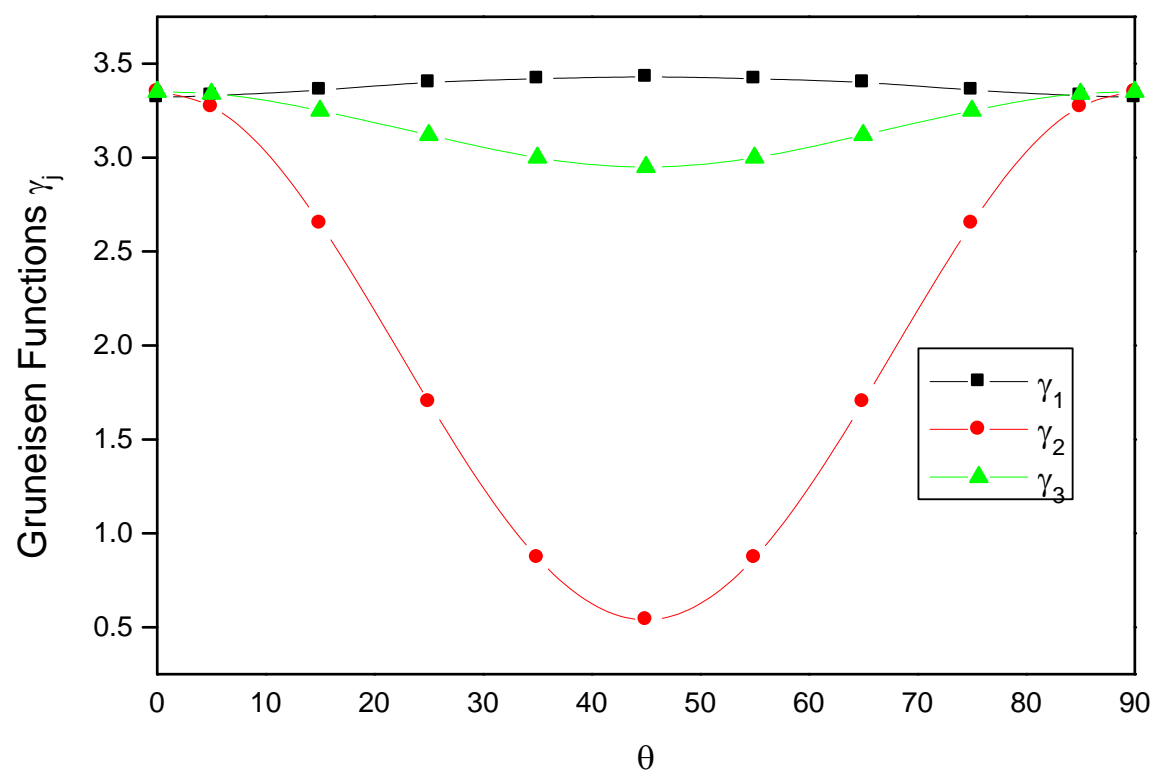

Figure 1: Variation of the Generalised Grüneisen functions $\gamma_{j}(\theta)$ as a function of angle $\theta$ of the $C_{60}$ system

\section{References}

[1] C. Kittel, 'Introduction to Solid State Physics', $7^{\text {th }}$ edition, John-Wiley and Sons, Inc Singapore.

[2] W. K. Fullagar, J. W. White, F. Trouw, Physica. B. 16, 213 (1995).

[3] R. V. Gopala Rao, R. Venkatesh, J. Phys. Chem. Solids. 62, 1923 (2001).

[4] M. Haluska, D. havlik, G. Kirlinger and W. Schranz, J. Phys. Condense. Matter. 11, 1009 (1999).

[5] N. P. Kobelev, R. K. Nikolaev, and Ya. M. Soifer, Physics of the Solid State. 43,2344 (2001).

[6] P. Espeau, B. Barrio, D. O. Lopez and H. Szware, Chem. Matter. 14, 321 (2002).

[7] L. T Scoh, M. M Boorum, B. J McMahon and A. de. Meijere, Science. 295, (2002).

[8] C. S. Sunder, R. Gupta, M. Premila and A.. K. Sood, J. Phys. Chem. Solids. 63, 1639 (2002).

[9] M. H. Manghnani, S. Tkachev, P. V. Zinin and I. A. Trojan, Phys. Rev. B. 64, 121403 (2001).

[10] H. Wang, C. Zeng, B. Wang and J. Yang, Phys. Rev. B. 63, 85417-1 (2001).

[11] Gerard Perrin, J. Phys. Chem. Solids. 62, 2091 (2001).

[12] Ya. M. Soifer, N. P. Kobelev and V. M. Levin, J. Alloys and Compounds. 319, 293 (2000).

[13]X-P. Li, J. P. Lu and R. M. Martin, Phys. Rev. B. 46, 4301 (1992).

[14] T. Yildirim and A. B. Harris, Phys. Rev. B. 46, 7878 (1992).

[15] Mary Anne White, G. MerinGasat, W. I. F. David and T. Mastno, Sol. Stat. Commn. 94, 481 (1995).

[16]F. D. Murnaghan, 'Finite Deformation of an Elastic Solid'. Wiley, New York, 1951

[17] Ramji Rao and Rajput, Phy.Stat.Sol. (b) 94 (1974), 691.

[18] K. Brugger and T. C. Fritz, Phys. Rev. A. 157, 524 (1967).

[19] C. S. Menon and R. Ramji Rao, J. Phys. Chem. Solids 33, 2129 (1972).

[20] R.N. Thruston and K. Brugger, Phys. Rev. A. 133, 1604 (1964).
[21] R. V. Gopala Rao and R. Venkatesh, J. Phys. Chem. Solids. 58, 939 (1997). 